\title{
Hypothesis on the distant spread of HER2-positive breast cancer brain metastasis via the human brain glymphatic system - Clinical and imaging data
}

\author{
Lena Marinova ${ }^{1 *}$, Radoslav Georgiev ${ }^{2}$ and Nikolay Evgeniev ${ }^{3}$ \\ ${ }^{1}$ Complex Oncology Center, Department of Radiotherapy; Russe, Bulgaria \\ "UMHAT”St.Marina”,Varna, Department of Imaging, Radiation therapy and Nuclear medicine; Varna, Bulgaria \\ ${ }^{3}$ Complex Oncology Center, Department of Medical oncology; Russe, Bulgaria
}

\begin{abstract}
Pre-clinical research in rodents provides evidence, that the central nervous system (CNS) has functional lymphatic vessels in 2012. A number of publications related to the study of glymphatic system in humans followed with the help of magnetic resonance imaging (MRI).

We present a 35-year-old woman who was diagnosed with invasive ductal carcinoma of the left mammary gland in 2011. The stage at diagnose was pT2N1M0, grade G3 with positive estrogen, progesterone and HER2 receptors expression. Complex treatment was carried out, including radical mastectomy with axillary dissection followed by adjuvant treatment- chemotherapy, radiotherapy, targeted therapy with trastuzumab and endocrine therapy with LHRH agonist plus tamoxifen.

In February 2019 a single brain metastasis has been found, extirpated and histologically veryfied. During May-June 2019 postoperative whole-brain radiotherapy (RT) to total dose (TD) 25 Gy was conducted with daily dose (DD) 2,5 Gy and boost in cerebral metastasis with brain ventricles to biologically equivalent TD 50 Gy. Against the background of complex treatment with 2 targeted agents including trastuzumab/pertuzumab and endocrine therapy with aromatase inhibitor, in July 2020 and followed by 18-Fluorodeoxyglucose (FDG)-PET/CT reports for metabolically active retrocrural and paraaortic lymph nodes.

Via the glymphatic brain system, for the first time, the hypothesis of metastatic brain cells transport to the paraaortic lymphatic vessels with metastasis formation in retrocrural and paraaortic lymph nodes is presented. The importance of imaging (CT scans, MRT and PET/CT) for the oncological diagnosis, stading, re-stading and disease progression is taken into account.
\end{abstract}

\section{Introduction}

Presentation of brain metastases in breast cancer patients is crucial event for expected poor outcomes, with a median survival that ranges from 2 to 25.3 months, despite treatment [1-3]. The presented clinical case with breast cancer and single brain metastasis after 8 years of complex treatment, created an extraordinary interest due to the manifested on PET/CT metabolically active retrocrural and paraaortic lymph nodes. A literary overview of the relationship between cerebral metastasis and metastatic paraaortic lymph nodes, showed that in 2015 , the traditional view of the brain having no lymphatic vessels was challenged by evidence showing functional lymphatic vessels lining the cranial dural sinuses in rodents [4,5]. Per Kristian Eide et al. show data on CNS lymphatic drainage to cervical lymph nodes in-vivo by magnetic resonance imaging (MRI) enhanced with an intrathecal contrast agent as a cerebrospinal fluid (CSF) tracer [6]. Studies using contrast-enhanced MRI were developed next and provided useful data to evaluate the glymphatic pathways $[7,8]$.

\section{Clinical case}

We present a 35-year-old woman who was diagnosed with invasive ductal carcinoma of the left mammary gland in 2011. The stage at diagnose was pT2N1M0, grade G3 with positive estrogen, progesterone and HER2 receptors expression. A radical mastectomy with axillary dissection, complex adjuvant treatment were conducted in the same year which included chemotherapy, radiotherapy, targeted therapy with trastuzumab and endocrine therapy with LHRH agonist plus tamoxifen. In 2015 extirpation of a left supraclavicular lymph node enlargement was performed with pathohistologically verification of lymph metastases from invasive ductal carcinoma. A multimodal treatment was conducted consisted of chemotherapy, bilateral adnexectomy, 2 targeted agents including trastuzumab/pertuzumab and endocrine therapy with aromatase inhibitor. In 2017 clinical remission has been achieved. In February 2019, a headache appeared, and on CT and MRT imaging data for solitary brain metastasis (Figures 1 and 2).

In April 2019, partial resection of cerebral metastasis was performed. Intraoperative: Line skin incision parasagital on the right, $5 \mathrm{~cm}$. front and $4 \mathrm{~cm}$. behind the skull coronal seam. Sinus sagithalis superior (SSS) is visualized. Dura mather opened arched with a base to the middle line. Multiple bridge veins draining to the SSS were found,

*Correspondence to: Lena Marinova, Complex Oncology Center, Department of Radiotherapy; Russe, Bulgaria, E-mail: rad_marinova@abv.bg

Key words: human brain glymphatic system, HER2-positive breast cancer, solitary brain metastasis, retrocrural and paraaortic lymph nodes, MRI, PET/CT, complex treatment

Received: August 19, 2020; Accepted: September 09, 2020; Published: September 14, 2020 

data

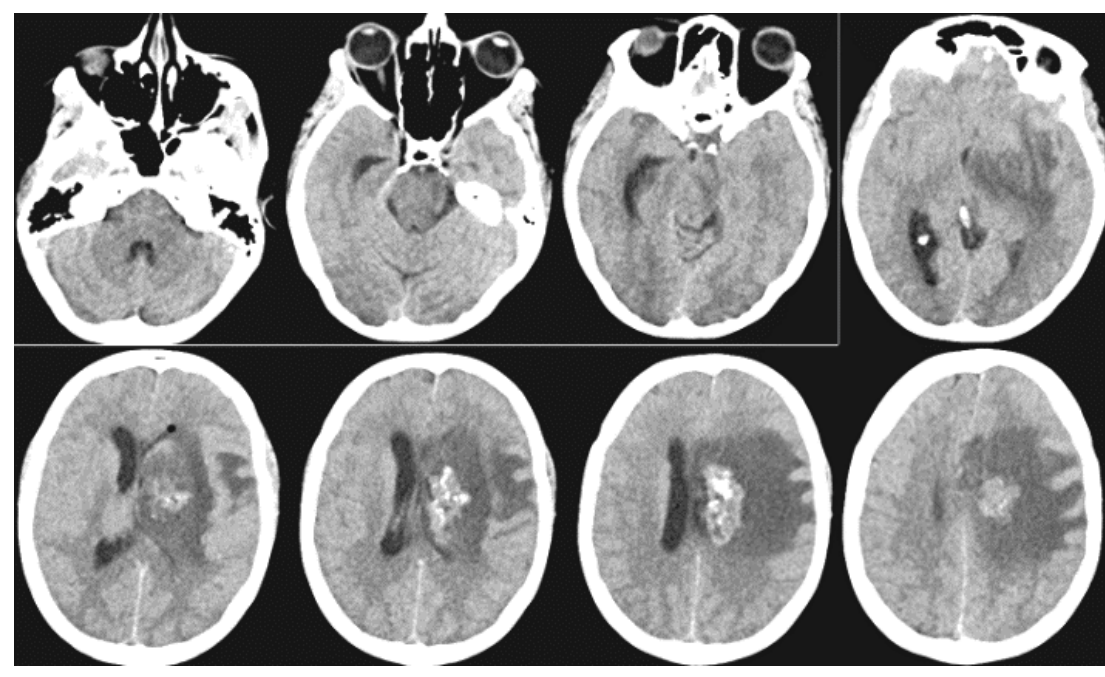

Figure1. Postcontrast CT of the brain / February 2019 - Heterogeneous contrast-enhancing periventricular mass with the presence of calcifications on the left frontal, with pronounced perifocal vasogenic edema and mass effect
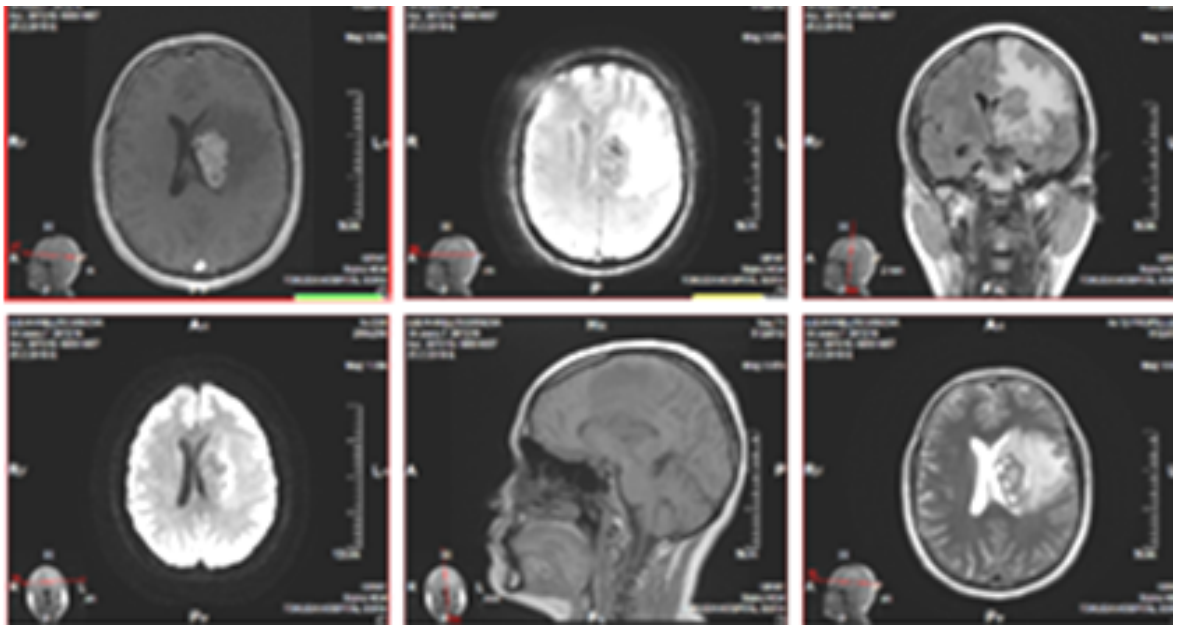

Figure 2. MRI of the brain / February 2019 - From left to right, top row: 3D T1 + C postcontrast, AX GRE T2, COR T2 FLAIR; bottom row: AX DWI, SAG T1, AX T2. Heterogeneous contrast-enhancing tumor mass periventricular left frontal, with cystic, probably necrotic areas, with calcifications and with pronounced perifocal vasogenic edema and mass effect

hampering interhemispheral access. The right frontal lobe is ecarded and in depth the two arteries cerebri anterior are dissected along the corpus callosum. A callosotomy was performed. It reached the left lateral ventriculum and located foramen Monroe. It came across an intraaxial tumor formation touching the lateral wall of the left lateral viticular. Partial tumor resection was performed. During the month May-June 2019 whole-brain radiotherapy (RT) was conducted up to total dose (TD) 25 Gy with daily dose (DD) 2.5 Gy and boost in brain metastasis and brain ventricles with DD 2 Gy up to biologically equivalent TD 50 Gy (Figure 3).

RT is combined with targeted therapy (Trastuzumab $600 \mathrm{mg}$. and Pertuzumab 840 mg.). Five months after RT, in November 2019, a control CT scan of the head, thorax and abdomen was performed. No pathological lymph nodes and distant metastases were reported, except for changes in the brain after RT (Figures 4 and 5).

Figures 6 and 7 show the control CT and MRI of the brain during the month March 2020

Against the background of complex treatment with Trastuzumab and Pertuzumab and endocrine therapy with aromatase inhibitor, PET/ CT during the July 2020 report metabolically active retrocrural and paraaortic lymph sites (Figure 8). Before RT of the paraaortic lymph chain, a CT scan of the brain was performed with evidence of increased cerebral edema compared to March 2020 (Figure 9).

\section{Discussion}

The glymphatic system is contemporarily one of the most studied functional systems of the human body [9-13]. In the presented clinical case, even before surgery by CT and MRT around the cerebral metastasis, a large perifocal vasogenic oedema with increased intracranial pressure in the left hemisphere is visualized (Figure 10). From the diffusion MRI marked with Gadolinium, the vasogenic edema is reported, which exerts pressure on the subarachnoid space and the soft dura mater.

Cerebral edema represents an increase in brain mass as a result of an increase in the water content of the brain parenchyma. Depending on the presence or absence of pathologies, two types of cerebral edema - vasogenous and cytotoxic - are distinguished. In the vasogenic type, there are violations in the barrier permeability (permeability of the vascular wall) with the passage of proteins into the extracellular spaces, an increase in osmotic pressure in the extracellular spaces to that of cell cytoplasms and the extraction of water from blood vessels. 


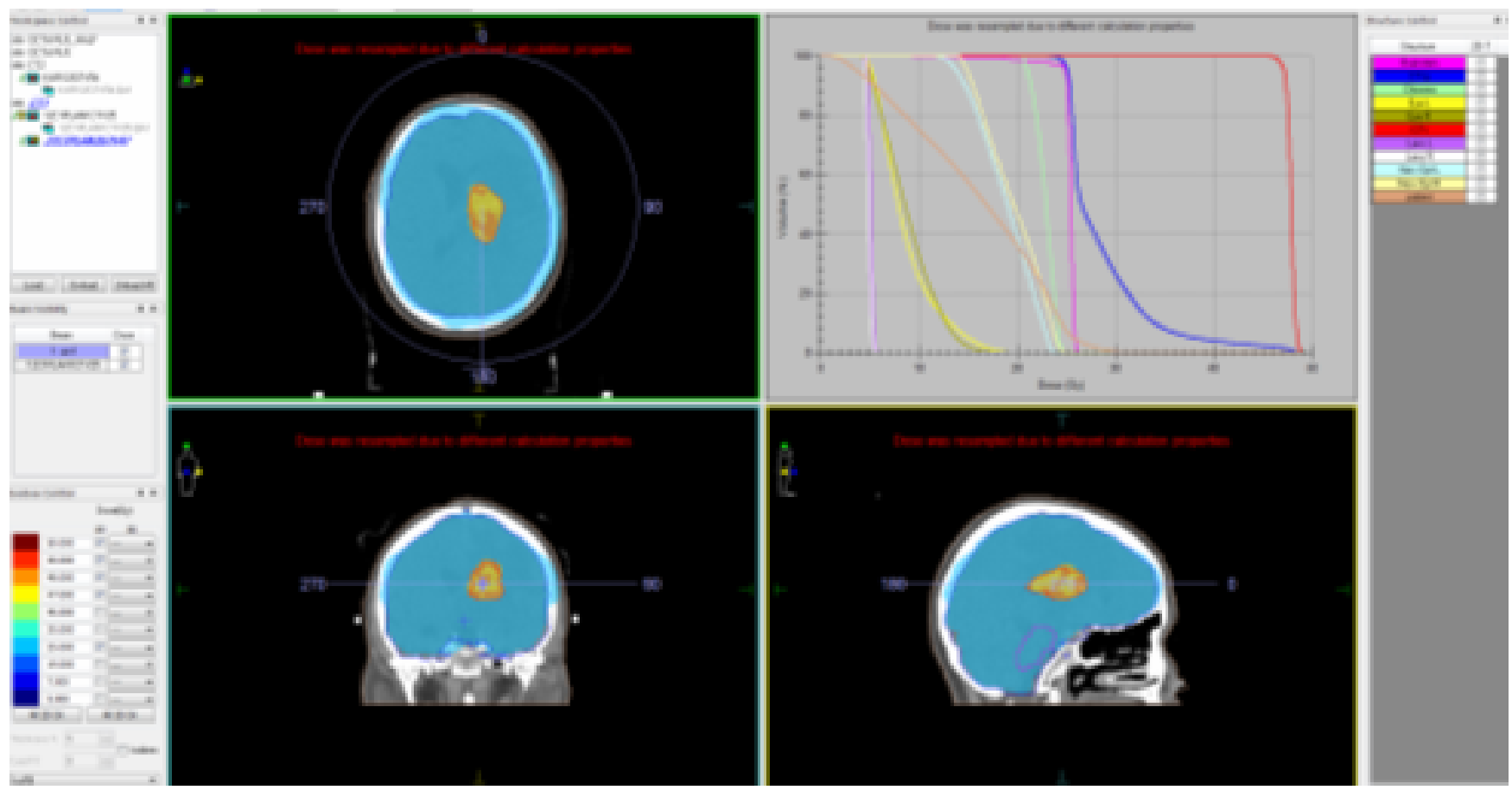

Figure 3. Whole-brain radiotherapy up to TD 25 Gy with DD 2.5 Gy and boost in brain metastasis and brain ventricles with DD 2 Gy up to biologically equivalent TD 50 Gy

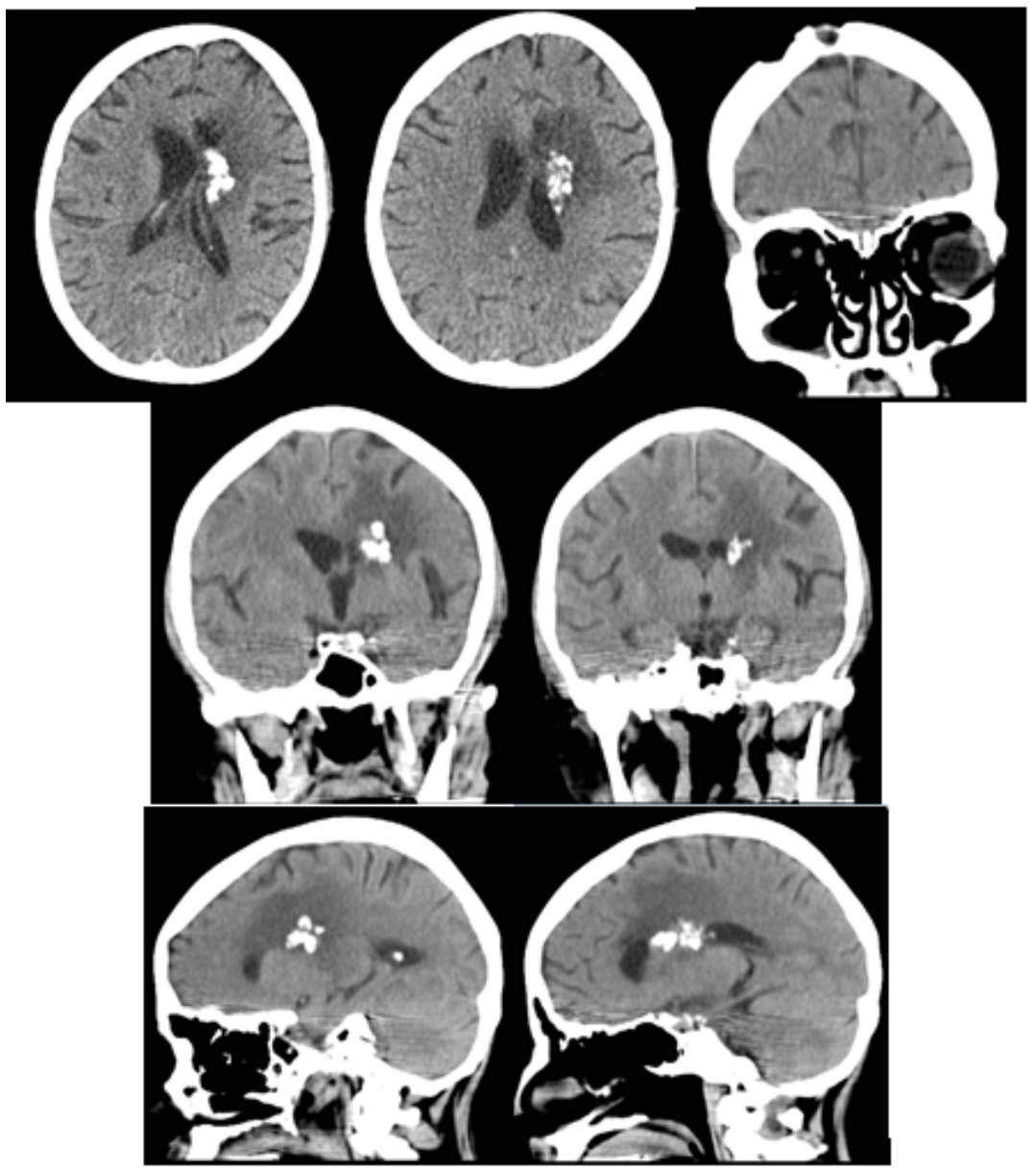

Figure 4. CT of the brain 5 months after radiotherapy / November 2019 -The formation is slightly reduced in size, there is a pronounced reduction in the mass effect and perifocal vasogenic edema on the left front 

data
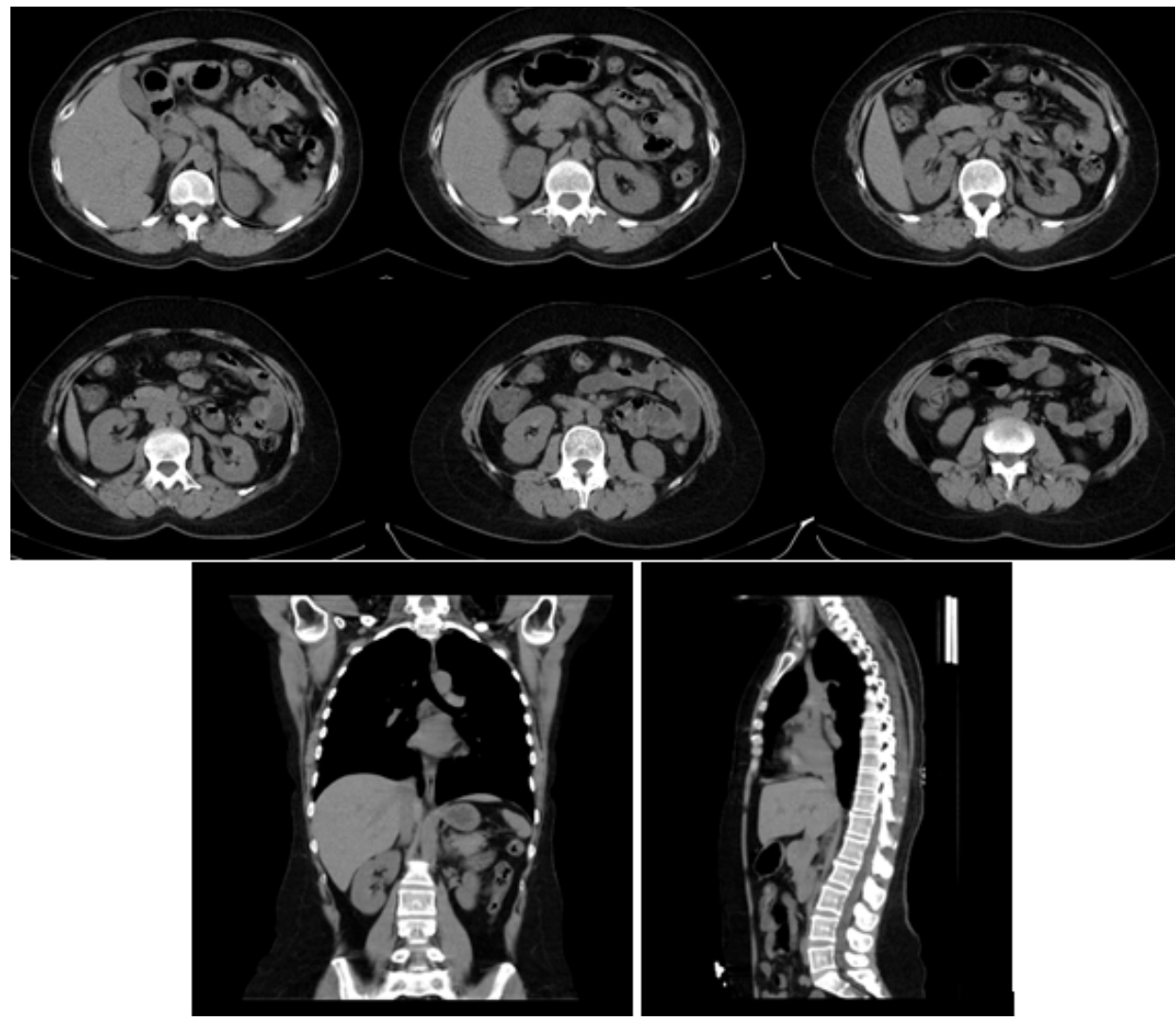

Figure 5. Axial CT of the abdomen, sagittal and coronary reconstructions of the abdomen and chest with a normal CT scan/ November 2019

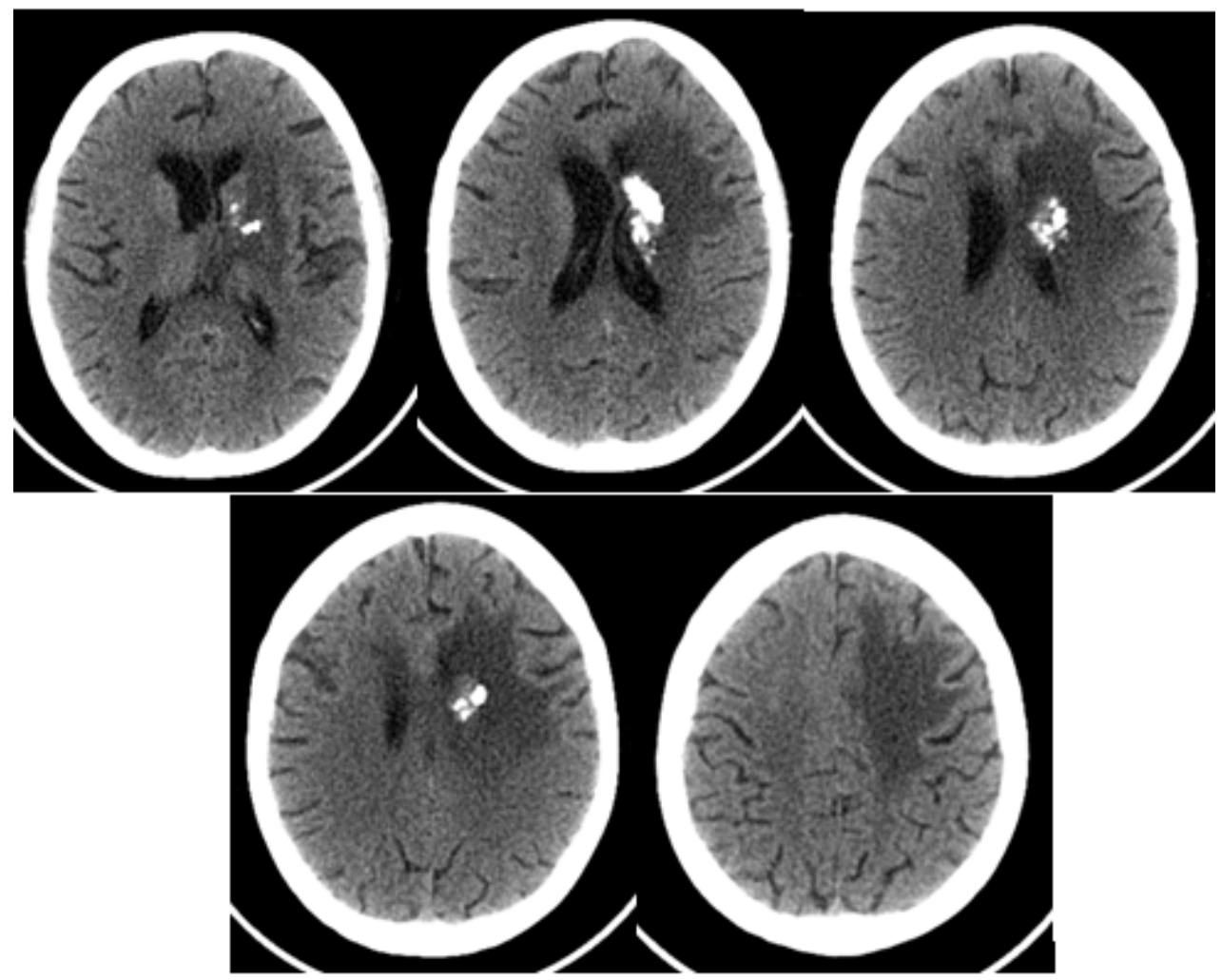

Figure 6. CT of the brain 9 months after radiotherapy / March 2020 - The formation is slightly reduced in size, there is a pronounced reduction of the mass effect and perifocal vasogenic edema to the left front, as well as an increase in calcifications in the lesion 

data

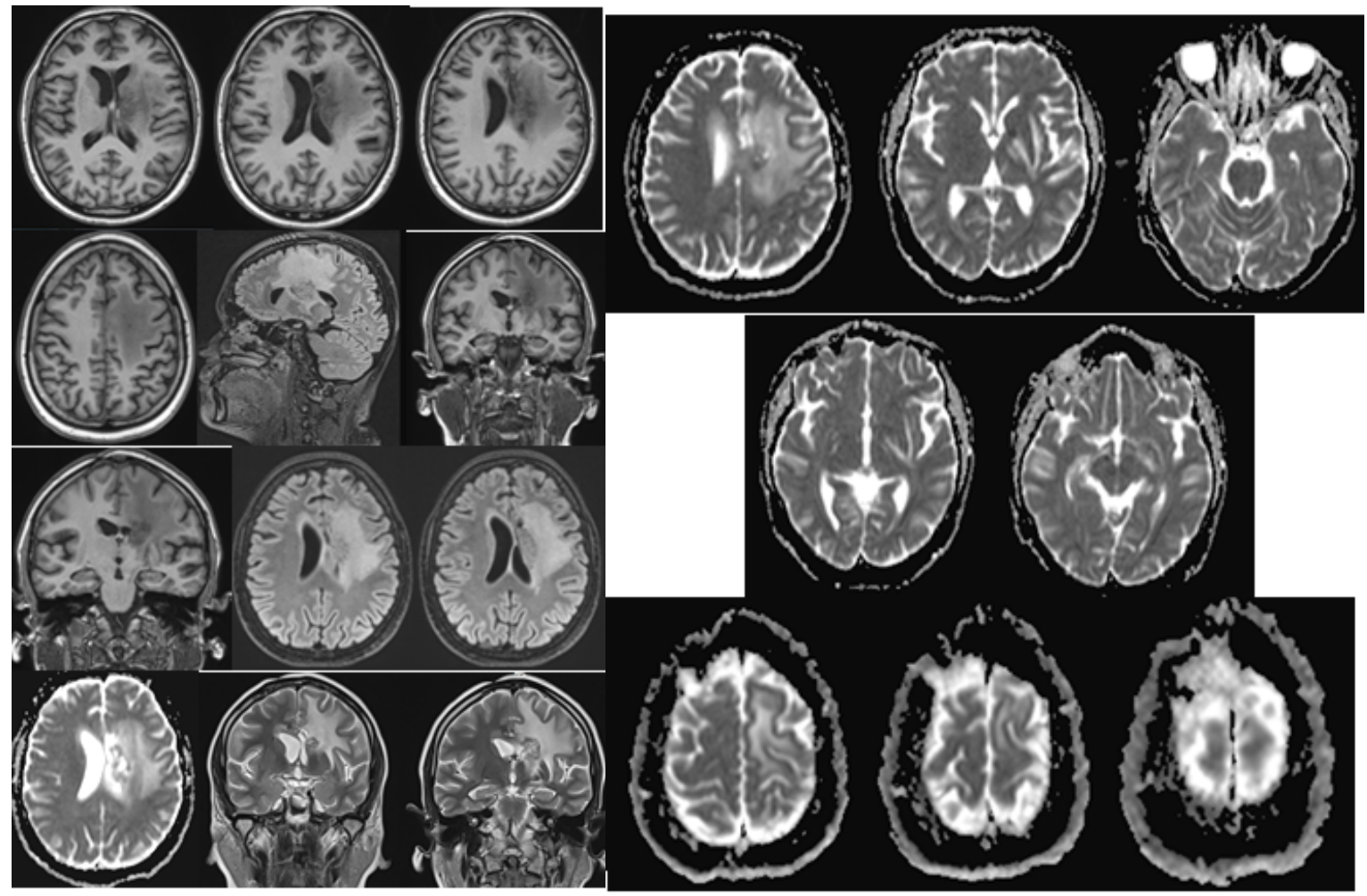

Figure 7. MR native images of the brain - axial 3DT1, axial T2 FLAIR, axial ADC, SAG T2 FLAIR / March 2020. No clear dynamics compared to the finding of Figure 3 of the CT in November 2019

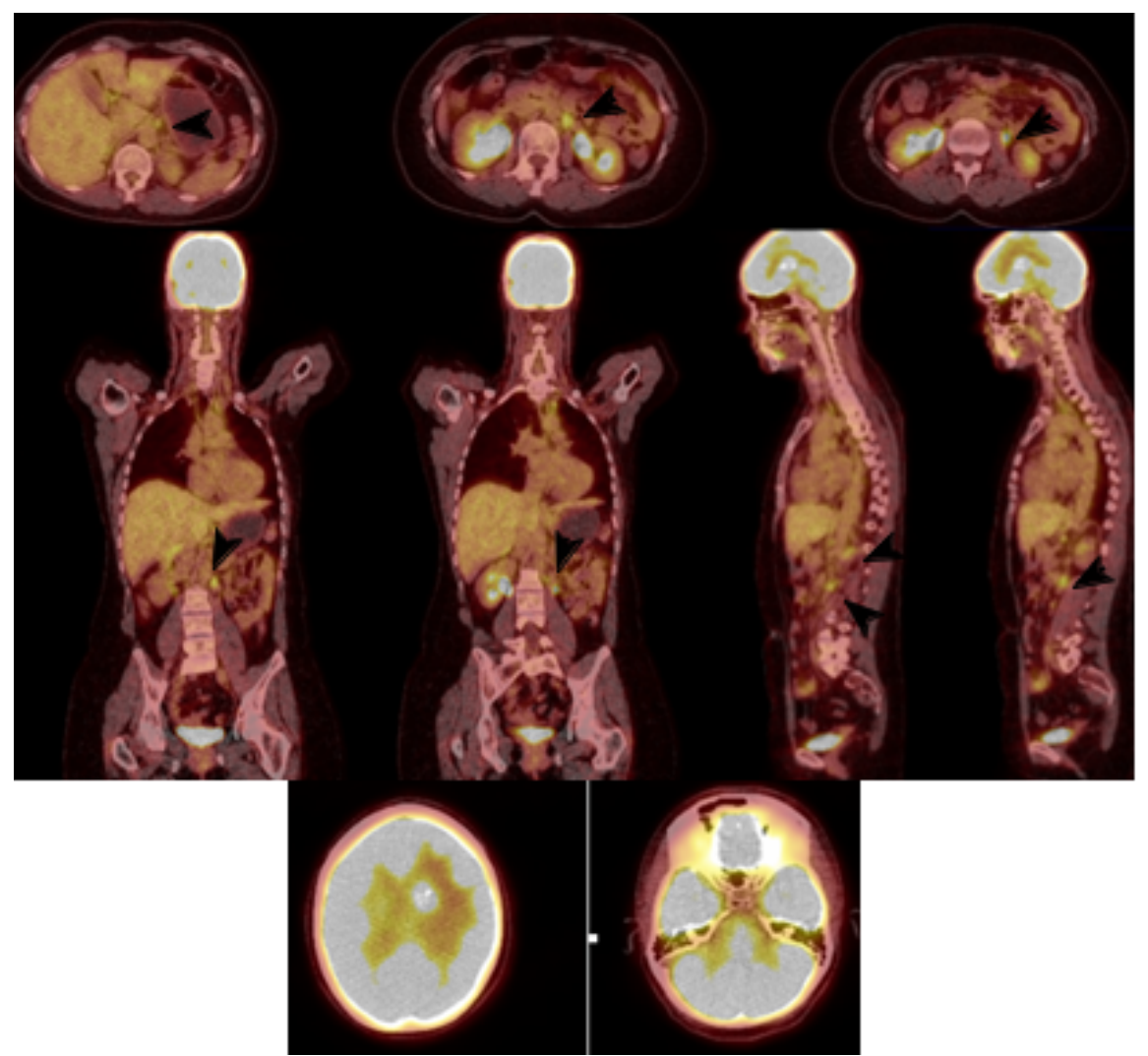

Figure 8. PET / CT axial sections of the abdomen, coronary and sagittal reconstructions (July 2020) detect metabolically active retrocrural and paraaortic lymph nodes, which are marked with arrows 


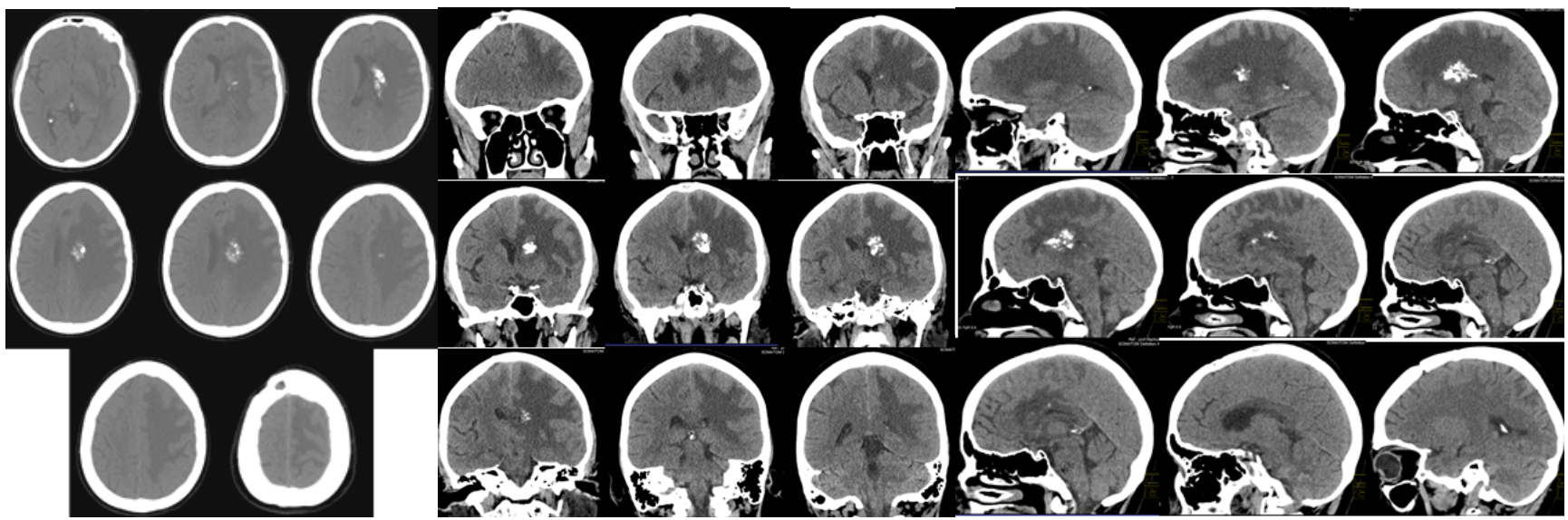

Figure 9. Compared to March 2020, the brain CT scan in August 2020 reported progression of vasogenic edema and mass effect left fronto-parietal, with coverage of new areas parietally and the appearance of compression and dislocation along the midline to the right

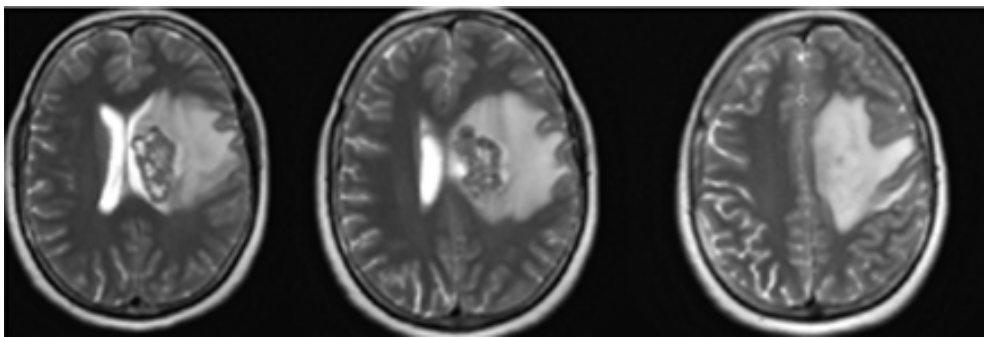

Figure 10. AX T2 MRI of the brain- Around the cerebral metastasis, a large perifocal vasogenic oedema with increased intracranial pressure in the left hemisphere / February 2019 before the operation and radiotherapy

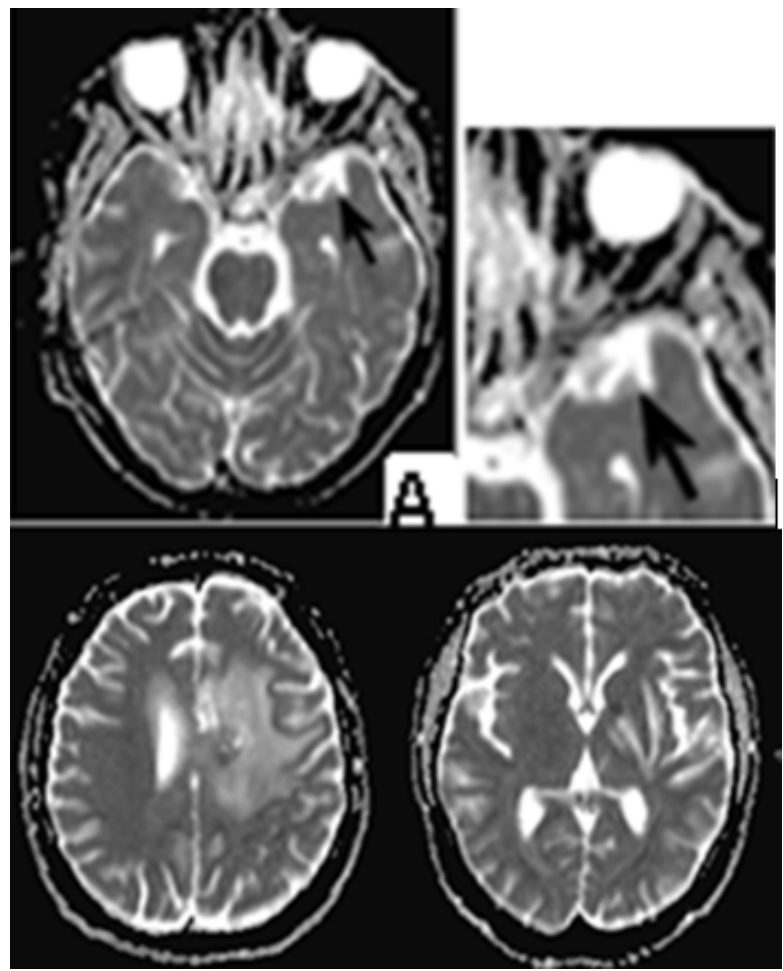

Figure 11. Diffusion weighted MRI/ axial T2 FLAIR - On the upper row on the left stands out a well-defined anterior temporal lobe perivascular space. MRI diffusion is facilitated. The sulci are slightly reduced due to the mass effect on the brains gyri, due to edema in the white matter on the left fronto-parietal 

data

Intraoperatively, multiple bridge veins are established, draining to sinus sagithalis superior, hindering intrahemispheric access. In the conditions of impaired blood-brain barrier and increased permeability of endothelial cells in the vascular wall, hydrostatic pressure becomes a determining factor for the extravasation of interstitial fluid (ISF) into the brain interstitium.

In primary brain tumors and brain metastases in the perifocal edema, there is a high probability of the presence of microscopic, diagnostically elusive multiple tumor cells. This requires the surrounding healthy area to be irradiated in malignant tumors, including that with vasogenic edema. Recent studies have demonstrated that CSF and interstitial fluid (ISF) continuously interchange. This exchange is facilitated by convective influx of CSF along the periarterial space [14].

The soft dura mater of the cerebral cortex tightly covers the surface of the brain. Its composition includes numerous blood vessels and bezos motor nerves. Around the blood vessels entering the brain, a perivascular space is formed, which is an extension of the subarachnoid space. It is formed from the wall of blood vessels, the part of the soft dura mater, that covers the vessel and the growths of the glial cells forming membrana limitans gliae. Perivascular spaces, also known as Virchow-Robin spaces, are pial-lined interstitial fluid-filled spaces in the brain that surround perforating vessels. They do not have a direct connection with the subarachnoid space and in fact, the fluid within them has a slightly different composition as compared to CSF. From the subarachnoid space, CSF is driven into the Virchow-Robin spaces by a combination of arterial pulsatility, respiration, and CSF pressure gradients and the loose fibrous matrix of the perivascular space can be viewed as a low resistance highway for CSF influx [15]. In pathological conditions, such as stroke, the innate inflammatory response and edema formation is initiated in the perivascular spaces [16]. From the perivascular space around which there is a cerebrospinal liquid, through the AQP4 water channels, the ISF is mixed with the perivenosis. The superficial cortical veins anastomose with the deep veins and empty into the superior sagittal sinus. Cerebral venous blood from the superior sagittal sinus and the deep veins leave the brain via a confluence of sinuses draining into the sigmoid sinuses and jugular veins [17].

CSF movement in the brain parenchyma drives the convexia of ISF to the perivenous spaces around large deep veins. In the absence of pathology, venous blood from the white brain matter is drained into the sinuses of the dura mater. However, at increased pressure, the perivenous ISF is not removed from the veins, but drained from the brain to the cervical lymphatic system through functional vessels / meningial tial vessels that are around the veins of the brain cortex [13].

Postoperative whole-brain radiotherapy to total dose (TD) 25 Gy was conducted to the particular patient with DD 2,5 Gy and boost in cerebral metastasis with brain ventricles to biologically equivalent TD $50 \mathrm{~Gy}$, which has an impact on the altered cellular permeabilitity of the soft dura mater and subarachnoid space. These two factors, the altered permeability of the vascular walls and increased pressure due to vasogenic edema are the cause of increased convexia of the ISF. Increased intracranial pressure is possible to change the type of convexia from layer (laminar) to turbulent and this affects the excretion of the tumor cell not via the venous system, but via the functional a glial "lymphatic" (glymphatic) paravascular pathway. Diffusion weighted magnetic resonance imaging (MRI) exploits the translational mobility of water molecules to obtain information on the microscopic behaviour of the tissues presence of macromolecules, presence and permeability of membranes, equilibrium intracellular-extracellular water [18]. In addition, the brain has adapted to use a unique paravascular route in which fluids may freely exchange between the brain ISF and the cerebrospinal fluid (CSF) along glial "lymphatic" (glymphatic) routes without crossing the tightly regulated endothelial cell (EC) layer [14]. After RT, diffusion weighted MRI has no restriction, on the contrary it is facilitated, typical in vasogenic edema. Sulcuses are slightly reduced due to the mass effect on the on the brains gyri, due to edema in the white matter on the left fronto-parietal (Figure 11).

This highly polarized macroscopic system of convective fluid fluxes with rapid interchange of CSF and interstitial fluid was entitled the glymphatic system based on its similarity to the lymphatic system in the peripheral tissue in function, and on the important role of glial AQP4 channels in the convective fluid transport [15]. The authors suggested that their findings of lymphatic vessels may represent the second step in the drainage of the interstitial fluid from the brain parenchyma into deep cervical lymph nodes after first been drained into the cerebrospinal fluid (CSF) through a glial "lymphatic" (glymphatic) paravascular pathway. The latter is suggested as a brain-wide route for clearance of water and waste solutes from the brain [14].

The question arises why the separated metastatic cell is located in the retroperitoneal lymph nodes, and not in the cervical or supraclavicular lymph nodes? While presenting the clinical case, we announced that in extirpation of a left supraclavicular lymph node enlargement with pathohistologically verified lymph metastases from invasive ductal carcinoma was performed in 2015 . We believe that the postoperative RT of regional lymph nodes (axillary, supra- and infraclavicular on the left) in 2011, as well as the operative intervention / extirpation of metastatic supraclavicular lymph nodes) has disrupted the lymphatic pathways, which causes metastasis to move retrocrurally and engage the paraaortic lymphatic chain.

For patients with HER2-positive breast cancer with solitary brain metastasis, consideration should be given to treat with combination of surgery, radiotherapy, chemotherapy and anti HER2 agents is at present the best way to extend the OS and improve the quality of life of such patients [19]. In the presented clinical case, multimodal cancer therapy (surgery, RT, chemotherapy and targeted therapy) was conducted. The particular subject is currently conducting RT on the paraaortic lymph nodes in combination with targeted therapy. Although the OS of patients with HER2-positive breast cancer has improved substantially in the trastuzumab (T-DM1) era [20-22], the incidence of brain metastases among these patients has been increasing in recent years. One of main reasons for this is that the blood brain barrier (BBB) makes the CNS a perfect sanctuary for tumor cells. The BBB is a barrier that selectively chooses molecules to enter the CNS. It consists of endothelial cells, a basement membrane, and astrocyte foot processes. The permeability of the BBB decreases 100-fold as the molecular weight of the drug increases from $200 \mathrm{Da}$ to $450 \mathrm{Da}$ [23]. Nadia Aalling Jessen quoted Yang et al. and remarks that "intrathecal lumbar injections, which are routinely used in clinical myelographic studies, provide a viable route to assess the basic parameters of glymphatic function" $[15,24]$. We believe that due to the continuous mixing of CSF and ISF, in clinical cases with brain metastases, intrathecal administration of targeted therapy is required in combination with local radiotherapy/radiosurgery. The goal is to overcome the blood-brain barrier and improve the healing effect by directly affecting metastatic tumor cells.

\section{Conclusions}

1. Via the glymphatic brain system, for the first time, the hypothesis of metastatic brain cells transport to the paraaortic lymphatic vessels 
with metastasis formation in retrocrural and paraaortic lymph nodes is presented;

2. The importance of imaging (CT scans, MRT and PET/CT) for the oncological diagnosis, stading, re-stading and disease progression is taken into account;

3. In HER2 positive breast cancers, late solitary brain metastasis require intrathecal administration of targeted therapy in combination with local radiotherapy/radiosurgery.

\section{References}

1. Leone JP, Lee AV, Brufsky AM (2015) Prognostic factors and survival of patients with brain metastasis from breast cancer who underwent craniotomy. Cancer Med 4: 989-94.

2. Ogawa K, Yoshii Y, Nishimaki T (2008) Treatment and prognosis of brain metastases from breast cancer. $J$ Neurooncol 86: 231-238.

3. Sperduto PW, Kased N, Roberge D (2012) Effect of tumor subtype on survival and the graded prognostic assessment for patients with breast cancer and brain metastases. Int J Radiat Oncol Biol Phys 82: 2111-2117. [Crossref]

4. Louveau A, Smirnov I, Keyes JT (2015) Structural and functional features of central nervous system lymphatic vessels. Nature 523: 337-341. [Crossref]

5. Aspelund A, Antila S, Proulx S (2015) A dural lymphatic vascular system that drains brain interstitial fluid and macromolecules. J Exp Med 212: 991-999. [Crossref]

6. Per Kristian Eide, Svein Are Sirirud Vatnehol, Kyrre Eeg Emblem, Geir Ringstad (2018) Magnetic resonance imaging provides evidence of glymphatic drainage from human brain to cervical lymph nodes. Scientific Reports 8: 7194.

7. Gaberel T, Gakuba C, Goulay R (2014) Impaired glymphatic perfusion after strokes revealed by contrast-enhanced MRI: a new target for fibrinolysis? Stroke 45(10): 3092 3096 .

8. Iliff JJ, Lee H, Yu M (2013) Brain-wide pathway for waste clearance captured by contrast-enhanced MRI. J Clin Invest 123(3): 99-309. [Crossref]

9. Jessen NA, Munk AS, Lundgaard I, Nedergaard M (2015) The Glymphatic System: Beginner's Guide. Neurochem Res 40(12): 2583-2599. [Crossref]

10. Nedergaard M (2013) Garbage truck of the brain. Science 340: 1529-1530. [Crossref]
11. Hui FK (2015) Clearing your mind: A glymphatic system? World Neurosurgery 83(5):715-717. [Crossref]

12. Ratner V, Gao Y, Lee H (2017) Cerebrospinal and interstitial fluid transport via the glymphatic pathway modeled by optimal mass transport. Neuroimage 152: 530-537. [Crossref]

13. Benveniste H, Lee H, Volkow ND (2017) The glymphatic pathway. Neuroscientist 1073858417691030

14. Iliff JJ, Wang M, Liao Y (2012) A paravascular pathway facilitates CSF flow through the brain parenchyma and the clearance of interstitial solutes, including amyloid beta. Science Translational Medicine 4: 147ra111. [Crossref]

15. Nadia Aalling Jessen, Anne Sofie Finmann Munk, Iben Lundgaard (2015) The Glymphatic System - A Beginner's Guide. Neurochem Res 40(12): 2583-2599. [Crossref]

16. Del Zoppo GJ, Moskowitz M, Nedergaard M (2015) The Neurovascular Unit and Responses to Ischemia.Stroke Pathophysiol. Diagnosis, Manag.

17. Cipolla M (2010) Anatomy and Ultrastructure. Cereb Circ .

18. R Luypaert, S Boujraf, S Sourbron, M Osteaux (2001) Diffusion and perfusion MRI: basic physics. European Journal of Radiotherapy 38(1): 19-27.

19. Nitesh Rohatgi, A. Munshi, P. Bajpai (2018) Practical consensus recommendations on Her2+ve breast cancer with solitary brain mets. South Asian J Cancer 7(2): 118-122. [Crossref]

20. Lin NU, Winer EP (2007) Brain metastases: The HER2 paradigm. Clin Cancer Res 13: $1648-1655$.

21. Pestalozzi BC, Holmes E, de Azambuja E (2013) CNS relapses in patients with HER2positive early breast cancer who have and have not received adjuvant trastuzumab: A retrospective substudy of the HERA trial (BIG 1-01). Lancet Oncol 14: 244-248. [Crossref]

22. Aversa C, Rossi V, Geuna E (2014) Metastatic breast cancer subtypes and central nervous system metastases. Breast 23: 623-628. [Crossref]

23. Koo T, Kim IA (2016) Brain metastasis in human epidermal growth factor recepto 2-positive breast cancer: From biology to treatment. Radiat Oncol J 34: 1-9. [Crossref]

24. 24.Yang L, Kress BT, Weber HJ (2013) Evaluating glymphatic pathway function utilizing clinically relevant intrathecal infusion of CSF tracer. $J$ Transl Med 11: 107 [Crossref]

Copyright: (C2020 Marinova L. This is an open-access article distributed under the terms of the Creative Commons Attribution License, which permits unrestricted use, distribution, and reproduction in any medium, provided the original author and source are credited. 